\title{
Improved Wireless Sensor Location Algorithm Based on Combined Particle Swarm-Quasi-Newton with Threshold N
}

\author{
https://doi.org/10.3991/ijoe.v14i05.8649 \\ Hongqiang Zhang( $\left.{ }^{\bowtie}\right)$, Chunhong Wang \\ Suihua University, Suihua, China \\ 23811251 @qq.com
}

\begin{abstract}
-this paper drills down and systematically analyzes the improved DV-Hop algorithm about the origin of its position errors. A new method is hereby given for modifying such position errors. Beyond that, the particle swarm-quasi-Newton algorithm is improved, intercepting the combined DVHop location algorithm according to the number of known nodes which has been defined by a threshold $\mathrm{N}$. The coordinate of unknown nodes is calculated and analyzed by the combined particle swarm-quasi-Newton algorithm. Then this paper makes a validation analysis on the results by the way of emulation. The results show that the improved algorithm is relatively superior with high precision and lower position error than improved DV-Hop as intercepted.
\end{abstract}

Keywords-DV-Hop location algorithm; localization algorithm; particle swarm-quasi-Newton; threshold N.

\section{$1 \quad$ Introduction}

With continuous advances in the science and technology, the wireless sensor network, as a brand new hotspot, has won the favor of more and more scholars [1], since it has been integrated into our lives more closely, ubiquitous everywhere. It's always there, and closely related to our daily life [2]. Nowadays, there are a lot of research areas about the wireless sensor network, of which the node localization algorithm has long been a hot topic since it is most basic and essential. It is therefore required to focus on the information elements of supervised object when data monitor analysis and radio communication are made, especially on the location of the observation point [3]. It makes no sense if the node position is undeterminable, so that it is vital for us to explore the node location [4].

In a broad sense, the wireless sensor is a means of access the information technology, where there is a huge mass of nodes at a relatively low price. The plenty of nodes are arranged in a predefined zone where they further make up a spontaneous multihop system. In doing so, it not only enables a cooperative sensing, but also acquire and process the information covered by all the perceived objects within the network coverage area, which is then aggregated and sent to the observer [5]. Three requisite 
elements are generally included in this phase, i.e. sensor, observer and object to be perceived [6]. In relation to the traditional wireless network, the wireless sensor network has a lower energy uncomplemented timely and the denser distribution of nodes [7]. Its network topology is susceptible to a variety of factors and subjected to change with them, so that it requires a higher level in respect of the wireless sensor network self-organization [8].

This paper explores and analyzes the DV-Hop algorithm, one of the localization algorithms for wireless sensor network. The combined particle swarm-quasi-Newton algorithm is applied for the improvement of DV-Hop algorithm as intercepted based on the number of known nodes, and is re-improved to a fine level. In the end, the particle swarm-quasi-Newton algorithm is integrated with improved DV-Hop algorithm organically for the purpose to make a simulation analysis and compare the results.on) [9].

\section{Analogue calculation based on the particle swarm and quasi- newton algorithms}

The particle swarm algorithm we discuss here is a kind of algorithm with adaptive analysis that auto operates in accordance with given conditions. It features simple design principle, easy to comprehend, and less parameters, however it is prone to convergence stagnation in the latter part [10]. For this reason, this paper proposes a type of algorithm to incorporate the strengths of particle swarm algorithm in the global search and local fine search of the quasi-Newton algorithm for the DV-Hop improvement in its localization algorithm, whereby to make simulation and analysis on the DV-Hop and two improved DV-Hops, respectively. In the end, the results are extracted from the simulation analysis for subsequent comprehensive and systematical investigation [11].

\subsection{Analysis of position error as the function of monitoring area}

Here we simulate how the primitive DV-Hop, the DV-Hops improved by the particle swarm algorithm and by the combined particle swarm-quasi-Newton algorithm are subjected to change with the monitoring areas, and then make a comparison on them [12].

1. Set the nodes of sensors distributed randomly to 100 , the number of known nodes to 10 , the node communication radius to $50 \mathrm{~m}$, and the side lengths of the monitoring area to $100 \mathrm{~m}, 150 \mathrm{~m}, 200 \mathrm{~m}, 250 \mathrm{~m}, 300 \mathrm{~m}$, respectively;

2. Set the nodes of sensors distributed randomly to 200 , the number of known nodes to 30 , the node communication radius to $50 \mathrm{~m}$, and the side lengths of the monitoring area to $100 \mathrm{~m}, 150 \mathrm{~m}, 200 \mathrm{~m}, 250 \mathrm{~m}, 300 \mathrm{~m}, 350 \mathrm{~m}, 400 \mathrm{~m}$, respectively;

As given in the above two cases, the plots changes in the average position errors of the above three algorithms available from the simulation experiment are shown in Fig. 2 and 3 . 


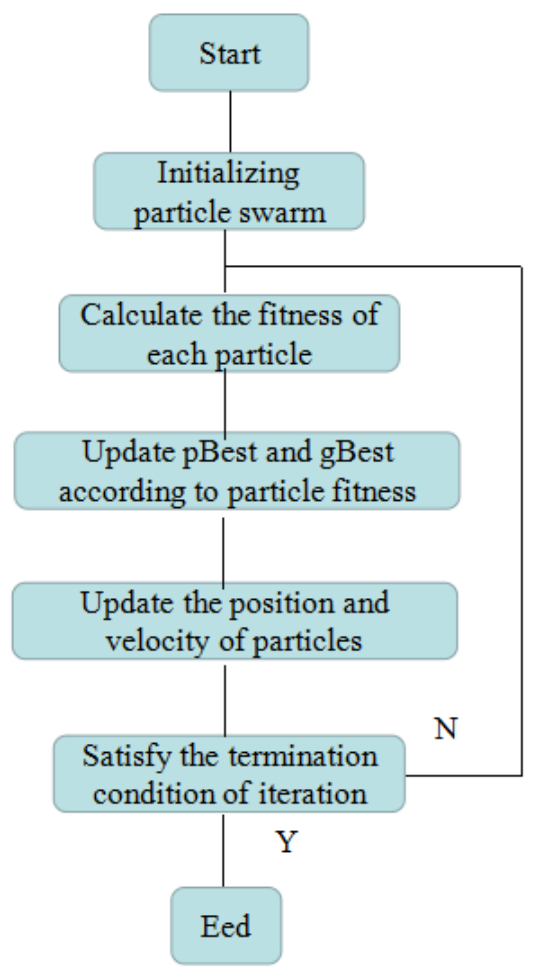

Fig. 1. Flow chart of particle swarm optimization algorithm

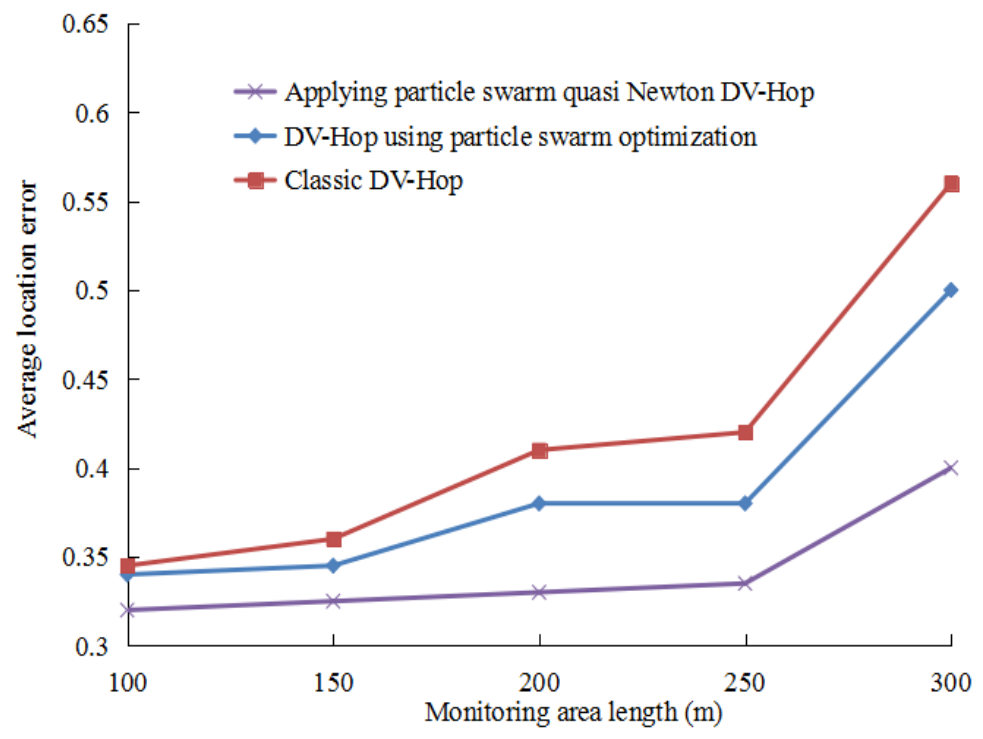

Fig. 2. Average position error under condition 1 


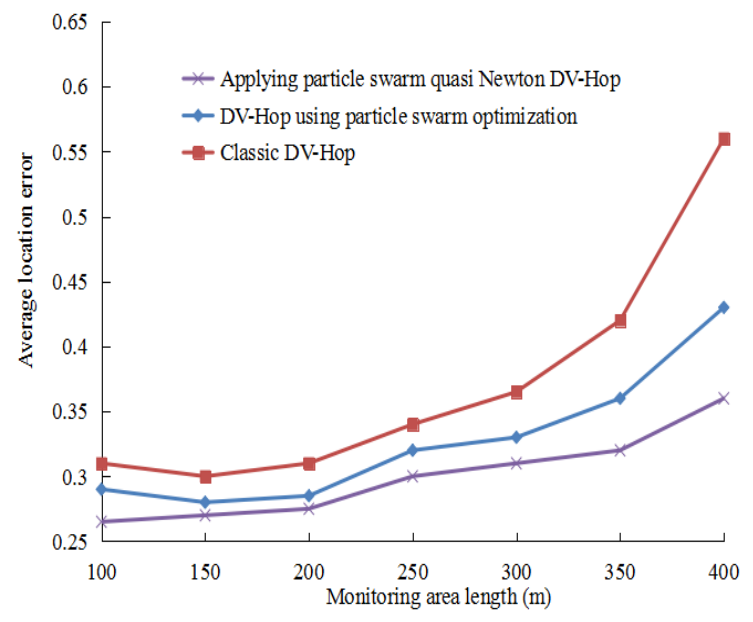

Fig. 3. Average position error under condition 2

As analyzed on the above curve, the trend of the overall change in the position errors tell us the position error of the DV-Hop improved based on particle swarm algorithm is less than that of the other two; the localization algorithm of DV-Hop improved based on the particle swarm-quasi-Newton algorithm gets a precision higher than that of the DV-Hop improved simply by the particle swarm algorithm. Comparative data from the emulation experiment reveals that the combined particle-quasiNewton algorithm has been proven to be highly effective in improving the location precision of DV-Hop, which exactly paraphrases the correct viewpoint of this paper.

\subsection{Simulation analysis of position errors as the function of sensor node communication radius}

The simulation analysis sets two different scenarios to simulate the change of the sensor node communication radius, whereby to compare the position errors of the three localization algorithms. The curve of comparing the average position error is available [13], and the evolution trends are shown in Fig. 4 and 5.

1. Set the coverage of monitoring area to $100 \times 100 \mathrm{~m}$, and the 100 sensor nodes as set are randomly distributed; there are total 10 known nodes with dynamic evolutions of communication radii assigned with values $20 \mathrm{~m}, 30 \mathrm{~m}, 40 \mathrm{~m}, 50 \mathrm{~m}$, respectively.

2. Set the coverage of monitoring area to $200 \times 200 \mathrm{~m}$, and the 200 sensor nodes as set are randomly distributed; there are total 20 known nodes with dynamic evolutions of communication radii assigned with values $30 \mathrm{~m}, 40 \mathrm{~m}, 50 \mathrm{~m}, 60 \mathrm{~m}, 70 \mathrm{~m}, 80 \mathrm{~m}$ respectively.

From the analysis of the evolution trends in the position errors, we can learn that when the node communication radius changes, the DV-Hop improved based on the combined particle swarm-quasi-Newton algorithm minimizes the position error when 
compared with the other two algorithms. In the three algorithms of simulation analysis, the average positioning error is the smallest, however, when the node communication radius grows to a certain value, it is not greatly improved in the term of the position error.

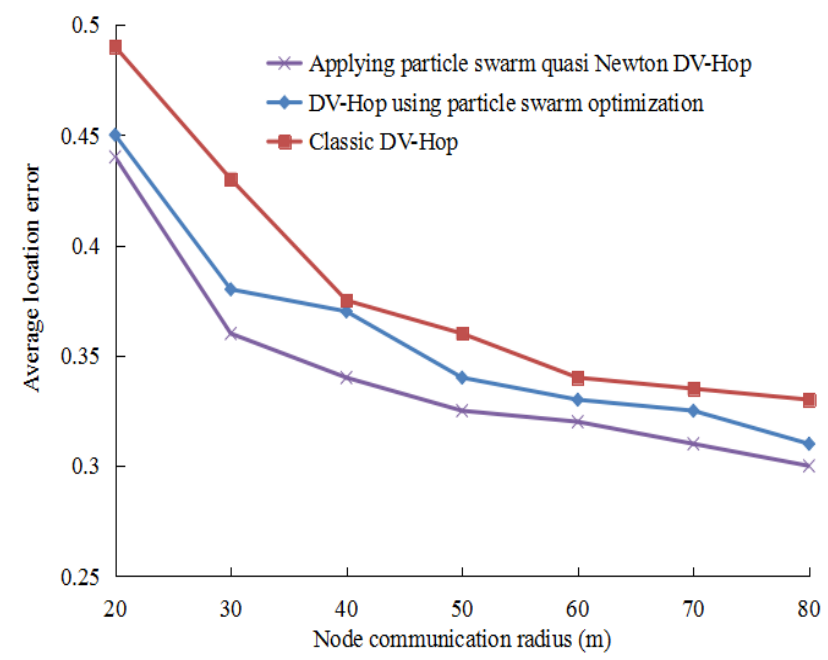

Fig. 4. Average positioning error under condition 1

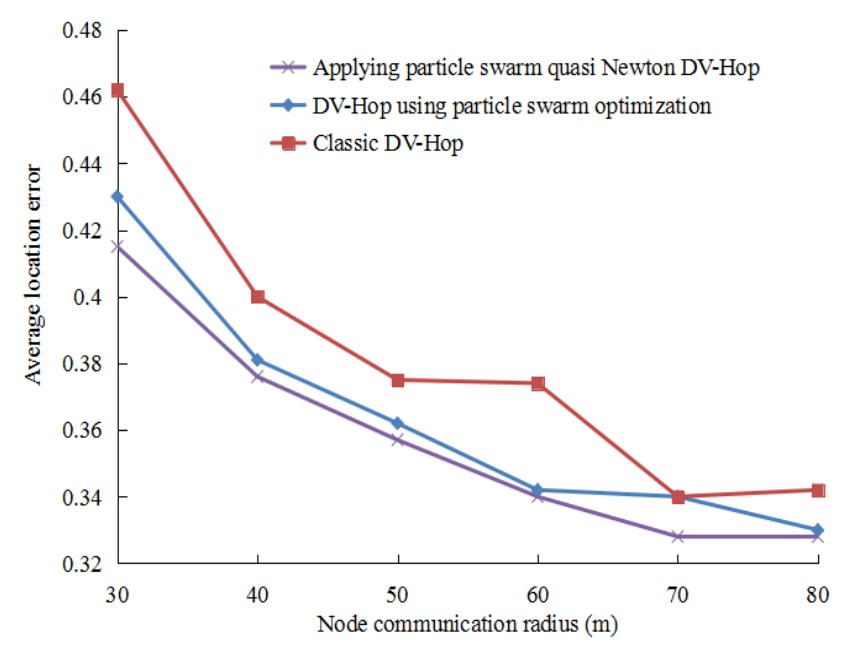

Fig. 5. Average positioning error under condition 2

\subsection{Analysis of position error as the function of node percentage}

These three position errors are compared and analyzed by simulating the change of the node percentage [14] with the results as shown in the Fig. 6 and 7. 
1. Set the coverage of monitoring area to $100 \times 100 \mathrm{~m}$, and the 100 sensor nodes as set are randomly distributed; set the node communication radii to $50 \mathrm{~m}$, and the number of nodes to $5,10,15,20,25$, respectively.

2. Set the coverage of monitoring area to $200 \times 200 \mathrm{~m}$, and the 200 sensor nodes as set are randomly distributed; set the node communication radii to $50 \mathrm{~m}$, and the number of nodes to $10,20 \mathrm{~m}, 30,40,50$, respectively.

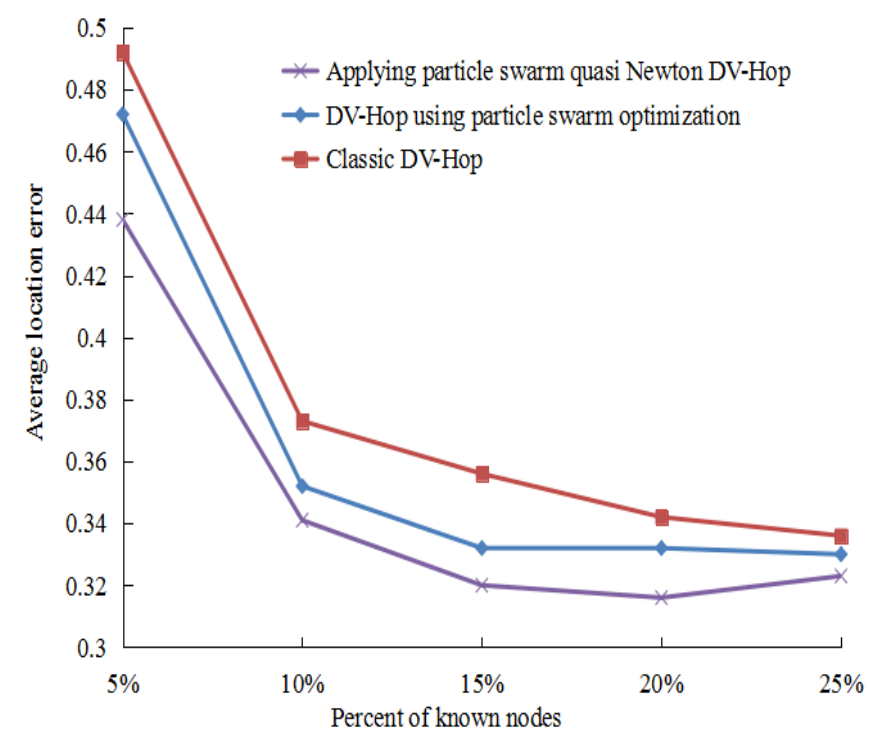

Fig. 6. Average position error under condition 1

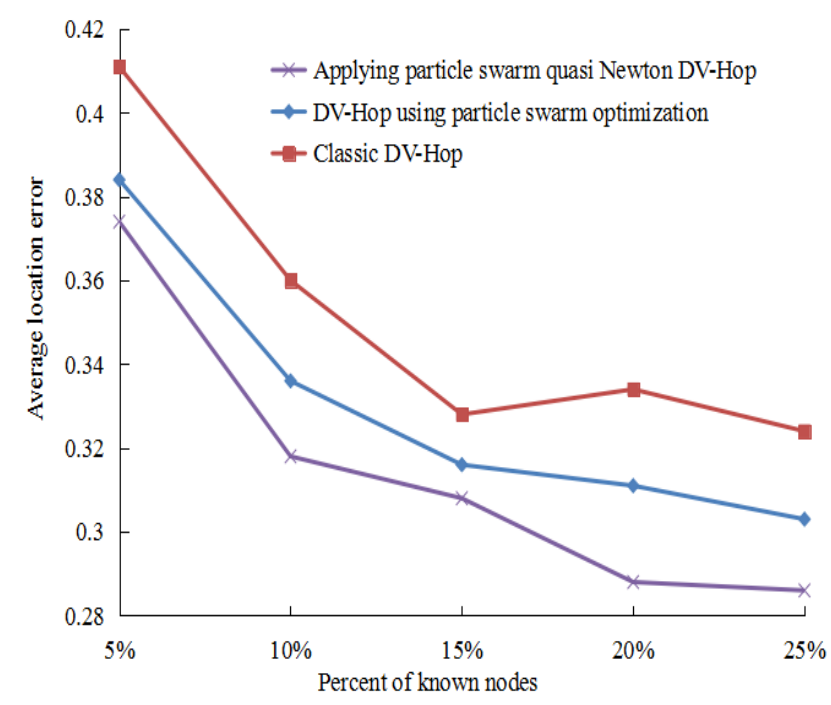

Fig. 7. Average position error under condition 2 
As seen from Figs. 6 and 7, that DV-Hop improved based on the combined particle swarm-quasi-Newton algorithm minimizes the position errors in relation to the other two algorithms, but somewhat less, maybe a few percent, even several tenths of percentage. Though tiny its precision is improved, this algorithm may also be considered in some applications occasions where the position precision is highly required.

\section{Investigation on the dv-hop_improved}

The population search enabled in the particle swarm algorithm is integrated with the local fine search function of the quasi-Newton algorithm to implement the further improvement of DV-Hop_improbed1 [15].

The unknown node coordinate is generally located by the following process:

1. First analyze the initial particle swarm;

2. The nonlinear equations can be formulated based on the distance between the unknown and known nodes as below:

$$
\left\{\begin{array}{l}
\sqrt{\left(x_{1}-x\right)^{2}+\left(y_{1}-y\right)^{2}}=d_{1} \\
\sqrt{\left(x_{n}-x\right)^{2}+\left(y_{n}-y\right)^{2}}=d_{n}
\end{array}\right.
$$

Suppose $f_{i}=\sqrt{\left(x_{i}-x\right)^{2}+\left(y_{i}-y\right)^{2}}$, and define the target function, i.e. a fitness function, as shown in the Equation 2:

$$
F=\sum_{i}\left(f_{i}-d_{i}\right)^{2}
$$

Where di represents the estimated distance between the unknown and known nodes.

\section{Dv-hop_improved2-based analogue simulation}

\subsection{Analogue simulation of position errors as the function of monitoring area}

1. Amidst the preset 100 sensor nodes as randomly distributed, set the number of known nodes to 10 , the node communication radius to $50 \mathrm{~m}$, the side lengths of monitoring area changes randomly, and the node communication radius changes dynamically, assign them the values 100. 150. 200. 250.300m.

2. Set the coverage of monitoring area to $200 \times 200 \mathrm{~m}$, the number of known nodes to 30 , the node communication radii to $50 \mathrm{~m}$, and the 200 sensor nodes as set are randomly distributed; the side lengths of monitoring area are stochastic to be $100 \mathrm{~m}$. $150 \mathrm{~m} .200 \mathrm{~m} .250 \mathrm{~m} .300 \mathrm{~m} .350 \mathrm{~m}$. $400 \mathrm{~m}$, respectively. 
As shown in the Fig. 8 and 9, the trend of plots change can be such that, when the monitoring area changes randomly, the DV-Hop_improved2 generates least position errors with dramatic improvement and a better calculation in relation to the other two algorithms. As the monitoring area extends, however, the position error also has the tendency to get bigger to be usual one.

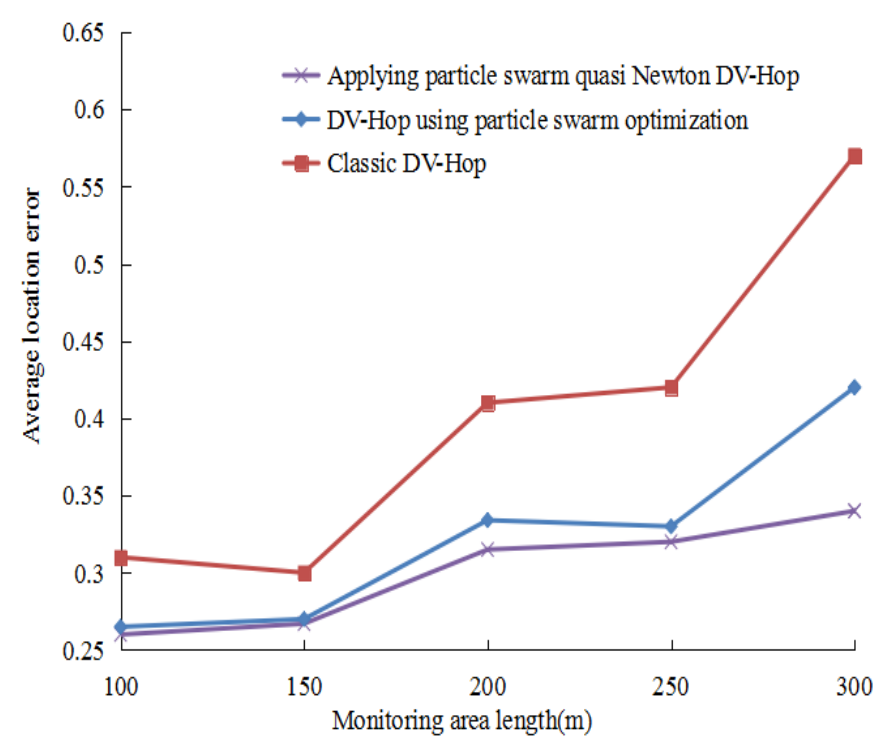

Fig. 8. Average position error under condition 1

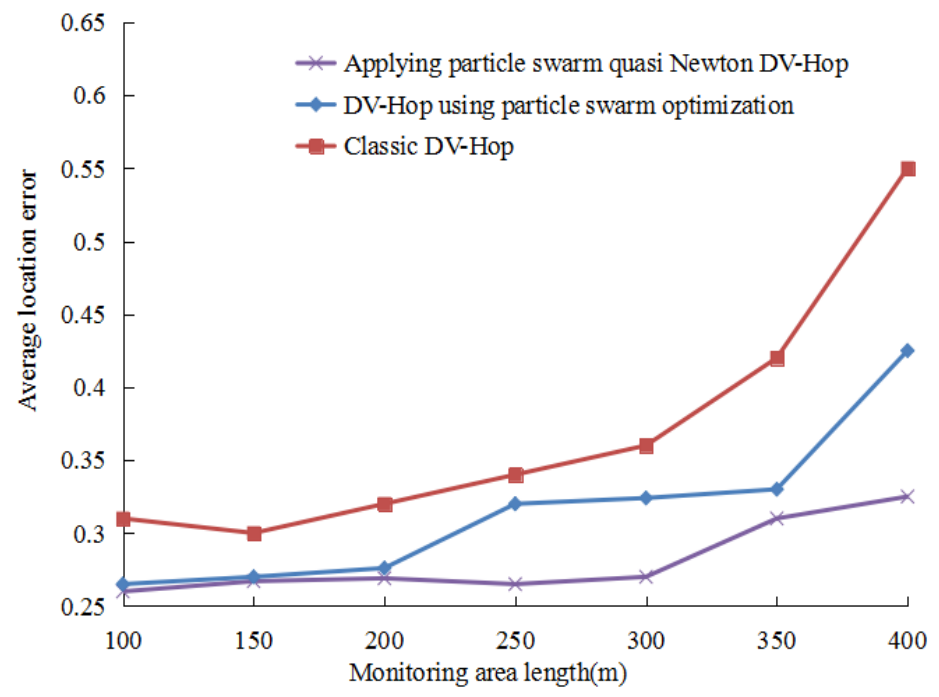

Fig. 9. Average position error under condition 2 


\subsection{Analysis of position error as the function of communication radius}

1. Set the coverage of monitoring area to $100 \times 100 \mathrm{~m}$, the 100 sensor nodes as set are randomly distributed; the number of known nodes is 30 , and the node communication radii are assigned with values $20 \mathrm{~m} .30 \mathrm{~m} .40 \mathrm{~m} .50 \mathrm{~m} .60 \mathrm{~m} .70 \mathrm{~m} .80 \mathrm{~m}$, respectively.

2. Set the coverage of monitoring area to $200 \times 200 \mathrm{~m}$, the 200 sensor nodes as set are randomly distributed; the number of known nodes is 10 , and the node communication radii change dynamically, assigned with values $30,40,50,60,70,80$, respectively.

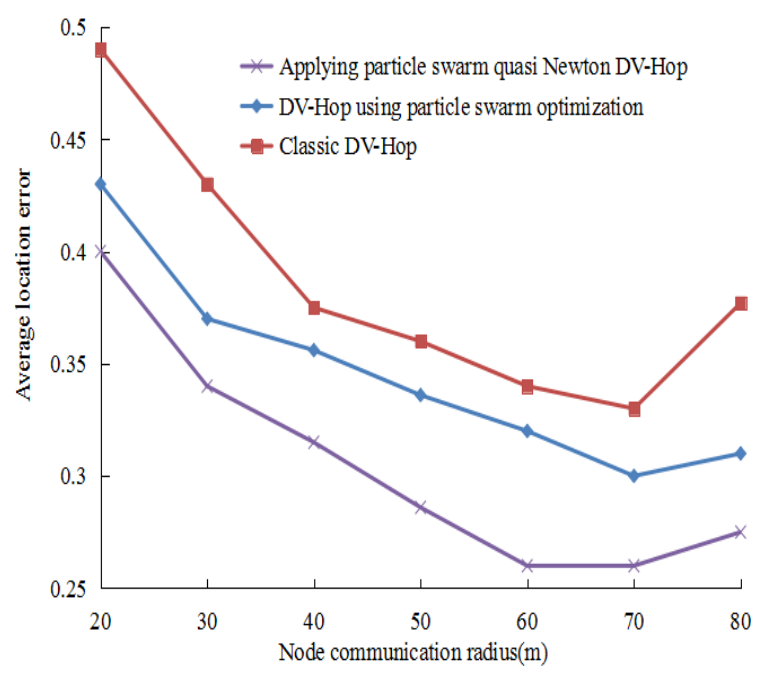

Fig. 10.Average position error under condition 1

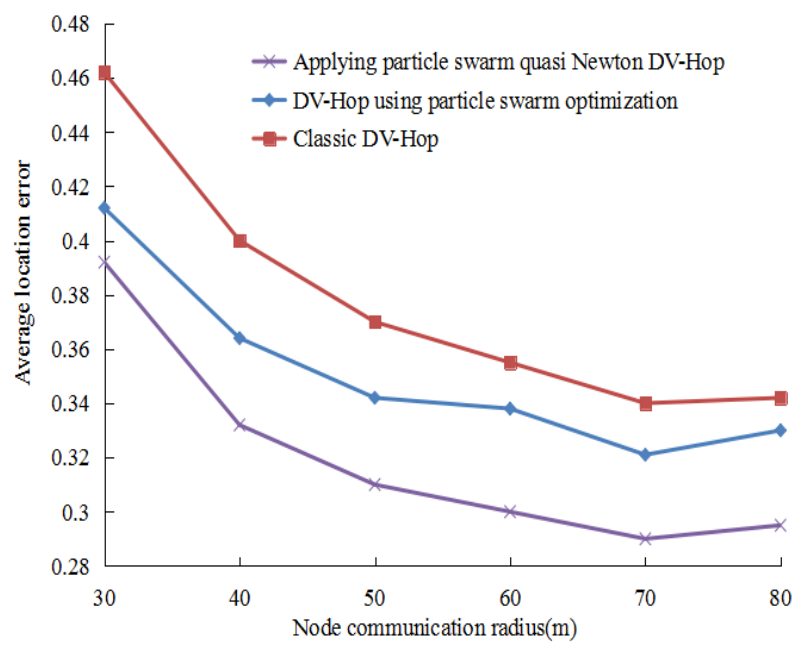

Fig. 11.Average position error under condition 2 
The results from simulation are shown in Fig. 10 and 11.

Analysis of plots change trend shown in Fig. 9 and 10 tells us that, compared with the traditional algorithm, the two methods show a great advantage, while the DVHop_improved 2 is particularly prominent since it has an obvious effect until the node communication radius reaches a certain value.

The results show that the combined particle swarm-quasi-Newton algorithm outperforms others in the term of location precision if applied to DV-Hop, and in conjunction with DV-Hop improved1, it can achieve a big improvement for algorithm. This paper integrates the selected DV-Hop with the particle swarm and quasi-Newton algorithms to systematically explore the general idea and basic process of the whole improved algorithm with better analysis results.

\section{Conclusion}

With reference to the numerous literature on the node location of wireless sensor network, this paper deeply and systematically explores the basic principle of DV-Hop algorithm about the origin of position errors. On this basis, the improved DV-Hop is proposed with a new idea for position errors. The conclusions are drawn as follows:

For the position errors occurred in DV-Hop's calculation process, a new idea is an integration of the particle swarm algorithm and the quasi-Newton algorithm that is designed for application in the location calculation process of the DV-Hop. The results from simulation analysis show that this integrated particle swarm-quasi-Newton algorithm minimizes the position errors of the improved DV-Hop with a better calculation effect.

The number of known nodes is defined with threshold N, by which the coordinates of the unknown nodes are calculated. Based on this process, the basic algorithm of particle swarm-quasi-Newton is used for simulation analysis. The results show that the improved DV-Hop based on the particle swarm-quasi-Newton algorithm outperforms the primitive algorithm with a minor error and a better effect.

\section{Acknowledgment}

Supported by Science and technology Fund of Heilongjiang Provincial Education Department: Upper computer serial communication design of stepper motor (2016KYYWF-0925); doctoral fund program at suihua college: Image quality evaluation of discrete imaging coupled system (SB11001)

\section{$7 \quad$ Reference}

[1] Gao Y., Zhao W. S., Jing, C., Ren W. Z. (2012). Wsn node localization algorithm based on adaptive particle swarm optimization. Applied Mechanics \& Materials, 1: 143-144. https://doi.org/10.4028/www.scientific.net/amm.143-144.302 
Paper-Improved Wireless Sensor Location Algorithm Based on Combined Particle Swarm-Quasi-Newt...

[2] Ren W., Zhao C. (2013). A localization algorithm based on sfla and pso for wireless sensor network. Information Technology Journal, 12: 502-505. https://doi.org/10.3923/itj. 2013.502.505

[3] LI X. Ming. (2013). Sensor network node localization based on improved dv-hop algorithm. Computer Simulation, 30: 281-284.

[4] Zhao J., Yi F. U., Wang H. B. (2013). Improved dv-hop localization algorithm for wireless sensor networks. Journal of Computer Applications, 7: 272-279. https://doi.org/10.3837/ tiis.2017.01.011

[5] Zhang Q., Cheng M. (2014). A node localization algorithm for wireless sensor network based on improved particle swarm optimization. Lecture Notes in Electrical Engineering, 237: 135-144. https://doi.org/10.1007/978-3-319-01273-5 14

[6] Zhang J., Wang H. B., Qiao X. G., Han Y. Q. (2014). Node localization algorithm based on dv-hop in wireless sensor networks. Applied Mechanics \& Materials, 440: 289-292.

[7] Zhao J., Fu. (2012). An improved dv-hop localisation algorithm based on particle swarm optimisation. Computer Applications \& Software, 29: 69-68. https://doi.org/10.1049/ cp. 2015.0868

[8] Jia S., Yang C. (2013). Sub-regional dv-hop localization algorithm for dynamic anchor nodes. International Journal of Applied Mathematics \& Statistics, 51: pp. 162-170.

[9] Chen X. Z., Liao M. H., Lin J. H. (2010). Improvement of node localization in wireless sensor network based on particle swarm optimization: improvement of node localization in wireless sensor network based on particle swarm optimization. Journal of Computer Applications, 30: 1736-1738. https://doi.org/10.3724/sp.j.1087.2010.01736

[10] Chen X., Zhang B. (2012). Improved dv-hop node localization algorithm in wireless sensor networks. International Journal of Distributed Sensor Networks, 2012: 1018-1020. https://doi.org/10.3837/tiis.2017.01.011

[11] Zhou T., Jiang F. (2013). Node localization of wireless sensor network based on mpso-dvhop. Computer Engineering \& Applications, 49: 52-55.

[12] Ye R., Zhao L. 2011. Localization algorithm for wireless sensor network bosed on acopso. Computer Measurement \& Control, 19: 732-735.

[13] Chen Z. G., Yi F. U., Wen-Bo X. U., Sun J. (2013). Swarm intelligence and mobile beacon improved dv-hop algorithm. Instrument Technique \& Sensor, 27: 112-115.

[14] Wang Y., Hu N. (2014). A novel particle swarm optimization based non-line of sight mobile node localization algorithm. Journal of Computational Information Systems, 10: 87598766.

[15] Shen Y. L., Zhang Y., Hu Y. P., Zhang J. (2015). A single chord localization algorithm for wireless sensor networks. International Journal of Grid \& Distributed Computing, 8: 14041406.https://doi.org/10.14257/ijgdc.2015.8.1.01

\section{Authors}

Zhang Hongqiang is an associate professor in College of Electrical Engineering, Suihua University, Suihua, China. His research interests include motor control, intelligent information processing, wireless sensor networks.

Wang Chunhong is a professor in College of Electrical Engineering, Suihua University, Suihua, China. His research interests include motor control, intelligent information processing.

Article submitted 30 March 2018. Final acceptance 05 May 2018. Final version published as submitted by the authors. 